\title{
Morfometria comparativa entre discos ópticos de familiares de glaucomatosos e normais pelo HRTII
}

\section{Heidelberg retina tomograph comparative measurements in relatives of glaucomatous patients and normal}

Alinne Maria Camargos da Costa ${ }^{1}$, Roberto Martins Gonçalves², Homero Gusmão de Almeida ${ }^{3}$, Sebastião Cronemberger ${ }^{4}$

\section{ResUMo}

Objetivo: Comparar discos ópticos de pacientes normais com história familiar de glaucoma primário de ângulo aberto (GPAA) com um grupo controle sem histórico familiar de glaucoma através da oftalmoscopia confocal a laser (HRTII). Métodos: Análise retrospectiva da morfometria dos discos ópticos de pacientes com e sem história familiar de GPAA. Cada paciente foi submetido a exame oftalmológico completo, perimetria computadorizada, paquimetria e HRTII. Os pacientes foram classificados em três grupos de acordo com o grau de parentesco: primeiro grau (grupo 1), segundo grau (grupo 2) e grupo controle sem história familiar de glaucoma (grupo 3). Foram analisados: área total do disco óptico (Área Total), área de faixa neural (FxN), área da escavação (Esc.), relação escavação/disco (E/D) e relação E/D linear (L). Para a análise estatística, utilizou-se o programa SPSS 12.0. considerando-se apenas um dos olhos de cada paciente selecionado aleatoriamente. Resultados: Foram incluídos setenta e quatro pacientes com idade média de 42,58 anos. Comparando-se os grupos 1 e 2, encontrou-se diferença estatisticamente significativa na variável Área Total, observando-se discos ópticos maiores no grupo 1. Comparando-se os grupos 1 e 3, as diferenças entre as variáveis Área Total, Esc, E/D e L foram estatisticamente significativas com predominância de valores mais elevados no grupo 1. Comparando-se os dados estereométricos entre os grupos $2 \mathrm{e}$ 3 não foram encontradas diferenças de valores estatisticamente significantes. Conclusão: Pacientes normais parentes de primeiro grau de glaucomatosos apresentam maiores valores das variáveis topográficas do disco óptico quando comparados aos dos pacientes sem histórico familiar de glaucoma.

Descritores: Glaucoma/diagnóstico; Fatores de risco; Nervo óptico; Oftalmoscopia;Topografia médica

\footnotetext{
'Pós-graduanda (Doutorado) em Oftalmologia da Faculdade de Medicina da UFMG - Belo Horizonte (MG) Brasil;

${ }^{2}$ Faculdade de Medicina da UFMG - Belo Horizonte (MG) Brasil;

${ }^{3}$ Faculdade de Medicina da UFMG - Belo Horizonte (MG) Brasil;

Serviço de Catarata e Glaucoma do Instituto de Olhos de Belo Horizonte (IOBH) - Belo Horizonte (MG) Brasil;

${ }^{4}$ Faculdade de Medicina da UFMG - Belo Horizonte (MG) Brasil.
}

Trabalho realizado no Instituto de Olhos de Belo Horizonte (IOBH) - Belo Horizonte (MG) Brasil.

Os autores declaram inexistir conflitos de interesses

Recebido para publicação em 12/1/2011 - Aceito para publicação em 26/6/2011 


\begin{abstract}
Purpose: To investigate whether there is a difference in topographic characteristics of optic nerve head analyzed by Heidelberg retina tomography (HRTII) in relatives of normal patients with or without history of open angle glaucoma. Methods: Retrospective study analyzing the optic disc morphometry of normal patients with or without family history of glaucoma. Each participant underwent a routine examination, visual field testing, pachimetry and optic nerve head topography. Patients were classified according their family history of glaucoma in groups as first-degree (group 1), far second-degree (group 2) and no siblings with glaucoma (control group). Optic disc analyzed parameters were: disc area, rim area, cup area, cup/disc ratio (E/D) and linear cup/disc ratio. Statistical analysis was made through the SPSS 12.0 program considering only one randomized eye. A $P$ value of less than 0.05 was considered statistically significant. Results: Seventy-four patients ( 74 eyes) were included. The average age was 42.58 years. In the stereometric analysis, the comparison between the disc area from the group 1 and group 2 was statistically significant $(\mathrm{P}<0.005)$, with marked overlap in group 1 , fact that was repeated for the variables disc area, cup area, cup/disc ratio linear cup/disc ratio comparing first -degree and control group. There was no statistical difference comparing group 2 and 3 in the stereometric analysis. Conclusion: First-degree siblings of glaucoma patients present stereometric differences in optic disc when compared with patients without history of glaucoma. These differences were found in patients with no signs of the disease.
\end{abstract}

\section{INTRODUÇÃO}

$\mathbf{0}$ glaucoma primário de ângulo aberto (GPAA) é uma das principais causas de cegueira irreversível em todo o mundo. Atualmente existem no Brasil cerca de 900.000 portadores da doença e acredita-se que, destes, 720.000 indivíduos são assintomáticos $^{(1)}$. Em países em desenvolvimento, $90 \%$ dos indivíduos afetados não sabem que possuem a doença $^{(2)}$ e, quando ficam cegos, não sabem o que causou sua cegueira $^{(2)}$. Considerando que a cegueira pelo GPAA é evitável quando essa doença é tratada nos seus estágios iniciais, o diagnóstico precoce é fundamental.

Sabe-se que dentre os fatores de risco para GPAA estão a pressão intraocular acima de $21 \mathrm{mmHg}$, raça, idade e história familiar. Estudos de coorte sugerem que mais de $50 \%$ de todos os glaucomas é familiar e uma história positiva para glaucoma confere um aumento superior a três vezes no risco de desenvolver GPAA ${ }^{(3-4)}$. O Rotterdam Eye Study investigou a agregação familiar de GPAA e encontrou que parentes de primeiro grau de pacientes com glaucoma apresentavam risco de $22 \%$ de desenvolver a doença em relação aos $2,3 \%$ de parentes dos controles normais ${ }^{(5)}$.

Há poucos dados na literatura a respeito da similaridade familiar da morfologia do disco óptico, mas alguns estudos indicam que existem determinantes genéticos na quantidade de células ganglionares em ratos, o que poderia afetar a morfologia do disco naquela espécie ${ }^{(6)}$.

A oftalmoscopia confocal a laser - Heidelberg Retina Tomograph (HRT) é um método de imagem da cabeça do disco óptico já disponível comercialmente há aproximadamente 15 anos. O software operacional está em contínua evolução com boa discriminação entre olhos normais e glaucomatosos e acompanhamento da progressão da doença ${ }^{(7)}$.

O objetivo deste estudo foi comparar através do HRTII as medidas topográficas dos discos ópticos de pacientes normais com história familiar de glaucoma primário de ângulo aberto com um grupo controle sem histórico familiar de glaucoma.

\section{Métodos}

Foi realizado um estudo retrospectivo, aprovado pelo Comitê de Ética em Pesquisa da Universidade Federal de Minas Gerais (COEP/UFMG).

Foram avaliados 74 prontuários de consultas ambulatoriais de rotina do Instituto de Olhos de Belo Horizonte, sendo 35 indivíduos portadores de história familiar positiva para glaucoma (segundo dados da anamnese) comparados com um grupo controle de 39 pacientes que não apresentavam casos de glaucoma conhecidos na família. Para a análise estatística considerou-se apenas um dos olhos selecionado aleatoriamente. Cada paciente foi submetido ao exame oftalmológico de rotina, além de paquimetria, perimetria computadorizada (Sita Standart Full Threshold programa 24-2, Humphrey Visual Field Analyzer, ZeissHumphrey Systems, Dublin, CA) e topografia dos discos ópticos (TDO) HRTII (Heildelberg Engineering, German). 


\section{Gráfico 1}

\section{Pressão intraocular média encontrada em familiares de glaucomatosos comparada ao grupo controle}

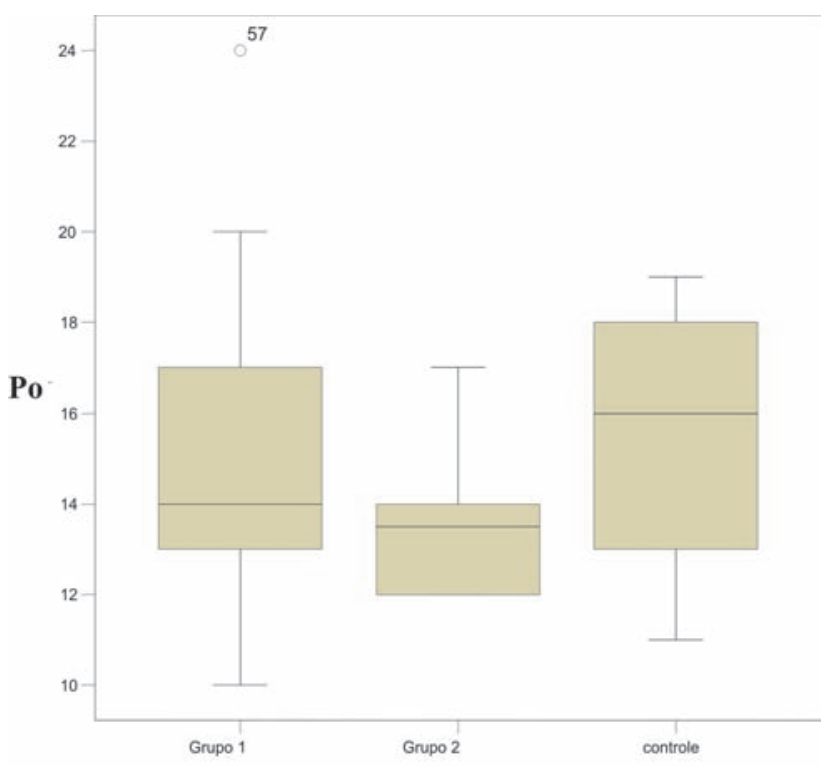

\section{Gráfico 3}

Índice global PSD do campo visual nos diversos grupos

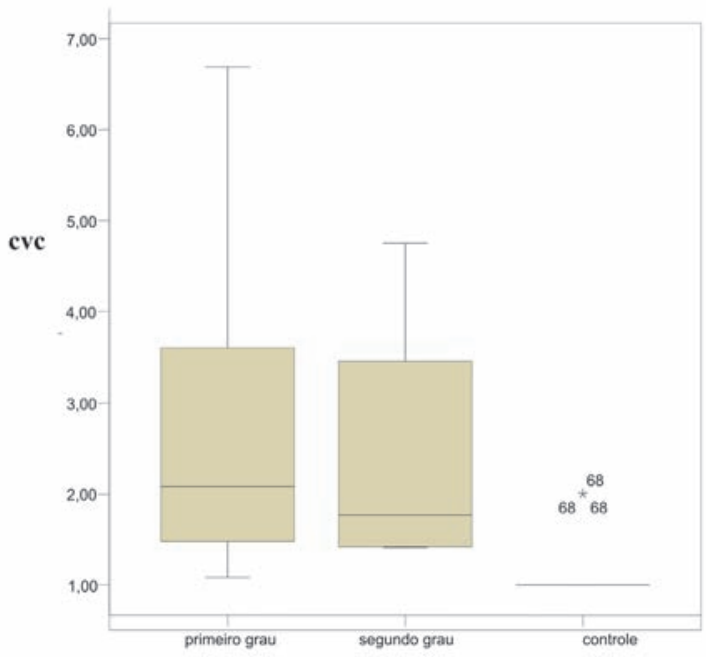

Gráfico 2

Paquimetria nos diversos grupos

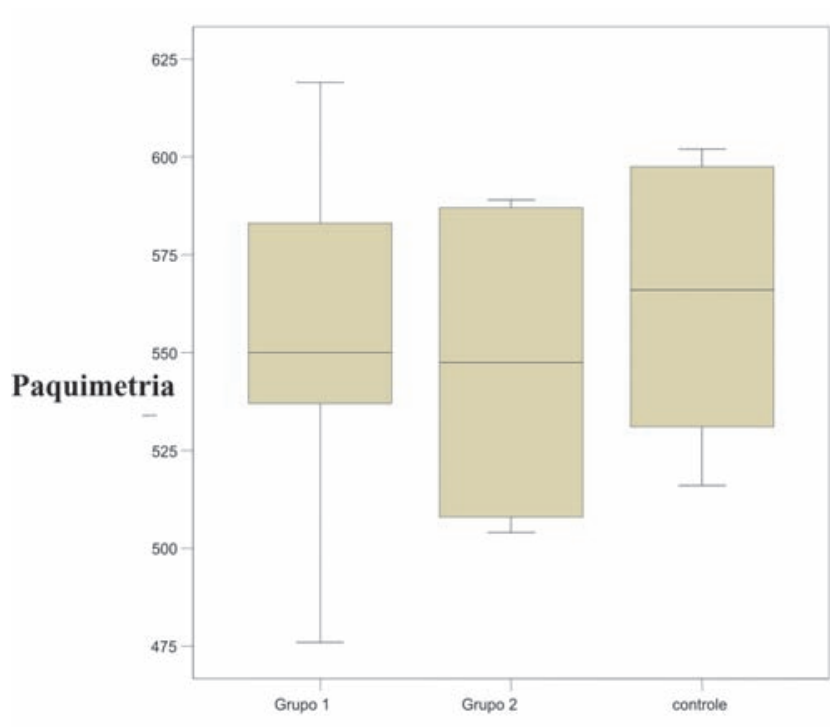

O HRTII foi realizado em pacientes com pupila dinâmica, por examinador experiente na aquisição das imagens topográficas. A marcação dos limites do disco óptico foi realizada pelo mesmo examinador que obteve as imagens topográficas. A imagem obtida pela média das três imagens topográficas simples, denominada imagem topográfica média, era utilizada para determinação dos limites do disco óptico. O aparelho HRT II então calculava os parâmetros do disco óptico.

Foram analisados os seguintes parâmetros obtidos com HRTII: área total do disco óptico (Área Total), área de faixa neural (FxN), área da escavação (Esc.), relação escavação/disco (E/D) e relação E/D linear (L). Para a análise estereométrica foram consideradas apenas as imagens com desvio padrão abaixo de $30 \mu \mathrm{m}$.

Os pacientes foram divididos em três grupos de acordo com o grau de parentesco com portadores da doença: Grupo 1- parentes de primeiro grau $(n=25)$; Grupo 2 - parentes de segundo grau $(\mathrm{n}=10)$ e Grupo controle - sem história familiar de glaucoma $(n=39)$.

Para a análise estatística, utilizou-se o programa SPSS 12.0. O teste t de Student para amostras independentes foi aplicado para comparação entre os grupos, com intervalo de confiança de $95 \%(\mathrm{p}<0,05)$. 
Tabela 1

Médias e desvios padrões das variáveis estereométricas analisadas pelo HRTII

\begin{tabular}{lccc}
\hline Variável & $\begin{array}{c}\text { Grupo 1 } \\
(\mathbf{N = 2 5 )} \\
\text { Média/DP }\end{array}$ & $\begin{array}{c}\text { Grupo 2 } \\
\mathbf{( N = 1 0 )} \\
\text { Média/DP }\end{array}$ & $\begin{array}{c}\text { Controle } \\
\mathbf{( N = 3 9 )} \\
\text { Média/DP }\end{array}$ \\
\hline Área total & $2,31 \pm 0,45$ & $1,97 \pm 0,42$ & $2,04 \pm 0,35$ \\
Àrea da escavação & $0,78 \pm 0,41$ & $0,49 \pm 0,34$ & $0,51 \pm 0,33$ \\
Área da faixa neural & $1,51 \pm 0,32$ & $1,48 \pm 0,39$ & $1,52 \pm 0,19$ \\
Relação E/D & $0,33 \pm 0,14$ & $0,24 \pm 0,17$ & $0,23 \pm 0,13$ \\
Relação E/D linear & $0,56 \pm 0,13$ & $0,46 \pm 0,17$ & $0,47 \pm 0,16$ \\
\hline
\end{tabular}

Tabela 2

Comparação entre os grupos 1 e 2

\begin{tabular}{lcccc}
\hline Variável & $\begin{array}{c}\text { Grupo 1 } \\
\text { (N=25) } \\
\text { Média/DP }\end{array}$ & $\begin{array}{c}\text { Grupo 2 } \\
\text { (N=10) } \\
\text { Média/DP }\end{array}$ & t & p \\
\hline Área total & $2,31 \pm 0,45$ & $1,97 \pm 0,42$ & 2,04 & $0,049^{*}$ \\
Àrea da escavação & $0,78 \pm 0,41$ & $0,49 \pm 0,34$ & 1,96 & 0,058 \\
Área da faixa neural & $1,51 \pm 0,32$ & $1,48 \pm 0,39$ & 0,25 & 0,806 \\
Relação E/D & $0,33 \pm 0,14$ & $0,24 \pm 0,17$ & 1,68 & 0,104 \\
Relação E/D linear & $0,56 \pm 0,13$ & $0,46 \pm 0,17$ & 1,92 & 0,063 \\
\hline
\end{tabular}

${ }^{*} p<0,05$

Tabela 3

Comparação entre os grupos 1 e 3

\begin{tabular}{lcccc}
\hline Variável & $\begin{array}{c}\text { Grupo 1 } \\
\text { (N=25) } \\
\text { Média/DP }\end{array}$ & $\begin{array}{c}\text { Controle } \\
\mathbf{( N = 3 9 )} \\
\text { Média/DP }\end{array}$ & t & p \\
\hline Área total & $2,31 \pm 0,09$ & $2,04 \pm 0,35$ & 2,68 & $0,009^{*}$ \\
Àrea da escavação & $0,78 \pm 0,08$ & $0,51 \pm 0,33$ & 2,87 & $0,006^{*}$ \\
Área da faixa neural & $1,51 \pm 0,07$ & $1,52 \pm 0,19$ & $-0,05$ & 0,955 \\
Relação E/D & $0,33 \pm 0,03$ & $0,23 \pm 0,13$ & 2,82 & $0,006^{*}$ \\
Relação E/D linear & $0,56 \pm 0,03$ & $0,47 \pm 0,16$ & 2,48 & $0,016^{*}$ \\
\hline
\end{tabular}

* $p<0,05$

Tabela 4

Comparação entre os grupos 2 e 3

\begin{tabular}{lcccc}
\hline Variável & $\begin{array}{c}\text { Grupo 2 } \\
\text { (N=10) } \\
\text { Média/DP }\end{array}$ & $\begin{array}{c}\text { Controle } \\
\mathbf{( N = 3 9 )} \\
\text { Média/DP }\end{array}$ & t & p \\
\hline Área total & $1,97 \pm 0,42$ & $2,04 \pm 0,35$ & $-0,580$ & 0,565 \\
Àrea da escavação & $0,49 \pm 0,34$ & $0,51 \pm 0,33$ & $-0,174$ & 0,863 \\
Área da faixa neural & $1,48 \pm 0,39$ & $1,52 \pm 0,19$ & 0,409 & 0,685 \\
Relação E/D & $0,24 \pm 0,17$ & $0,23 \pm 0,13$ & $-0,088$ & 0,930 \\
Relação E/D linear & $0,46 \pm 0,17$ & $0,47 \pm 0,16$ & $-0,135$ & 0,893 \\
\hline
\end{tabular}




\section{Resultados}

A idade dos pacientes variou de 16 a 73 anos (média de 44,8 $\pm 13,1$ anos no grupo 1, média de $41,5 \pm 14,1$ anos no grupo 2 e média de 41,4 417,3 anos no grupo controle). Não houve diferença estatisticamente significativa entre os grupos na avaliação da variável idade. Quarenta $(54,1 \%)$ pacientes eram do sexo feminino e 34 (45,9\%) do sexo masculino, sendo que no grupo 1 houve predominância feminina (88\%) e, nos outros dois grupos, predominância masculina ( $60 \%$ no grupo 2 e $64 \%$ no controle). Os pacientes do grupo 1 apresentaram pressão intraocular média de $15,1 \pm 3,4 \mathrm{mmHg}$ e espessura corneana central de 476 a $619 \mu \mathrm{m}$ (média de 550,9 $\pm 38,2$ ). Os pacientes do grupo 2 apresentaram pressão intraocular que variou média de $13,7 \pm 1,9 \mathrm{mmHg}$ e espessura corneana central de 504 a $589 \mu$ m (média de $547,2 \pm 36,9$ ). O grupo controle apresentou pressão intraocular média de $15,5 \pm 2,8 \mathrm{mmHg}$ e espessura corneana central de 516 a

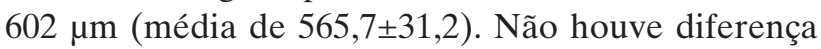
estatisticamente significante entre os valores pressóricos e paquimétricos na comparação entre os grupos (Gráficos 1 e 2). Os campos visuais apresentaram MD médio de $-0,96 \mathrm{~dB}$ no grupo $1,-1,05 \mathrm{~dB}$ no grupo 2 e - $0,44 \mathrm{~dB}$ no grupo controle. O PSD médio no grupo 1 foi de 2,69 dB, no grupo $2 \mathrm{de} 2,43 \mathrm{~dB}$ e no grupo controle de 1,17 dB.Diferença entre os grupos 1 e 3 foi considerada estatisticamente significativa no índice global PSD que foi mais elevado no grupo 1, fato esse que se repetiu na comparação entre os grupos 2 e 3 (Gráfico 3).

Com relação aos dados topográficos dos discos ópticos verificou-se que as medidas seguem a distribuição normal.

A tabela 1 lista as médias e os desvios padrões de todas as variáveis estereométricas analisadas para cada grupo.

Na comparação entre os grupos 1 e 2, as diferenças entre a variável área do disco, foram estatisticamente significantes, observando-se discos ópticos maiores em parentes de primeiro grau de pacientes glaucomatosos. Comparando-se os grupos 1 e 3 (controle) as diferenças entre as variáveis área do disco, área da escavação, relação E/D e relação E/D linear foram estatisticamente significativas com predominância de valores mais elevados no grupo 1 (Tabelas 2 e 3 ).

Comparando-se os dados estereométricos entre os grupos 2 e 3 , não foram encontradas diferenças estatisticamente significantes.

\section{DısCUSSÃO}

O glaucoma familiar é definido como a presença de um parente até de quarto grau (tataravô ou filhos de primos de primeiro grau) de uma pessoa afetada pela doença. Casos sem história familiar da doença são considerados esporádicos. Segundo alguns autores ${ }^{(3)}$, pacientes com glaucoma familiar apresentam maior gravidade e início mais precoce do glaucoma quando comparados com pacientes com glaucoma esporádico, o que implica em maior importância de serem rastreados e tratados.

Atualmente, a principal importância dos programas de rastreamento consiste na promoção do conhecimento sobre o risco de ser portador de glaucoma. Entretanto, apesar de várias entidades promoverem a iniciativa de programas de triagem em massa, um protocolo eficiente e custo-efetivo ainda não está disponível ${ }^{(8)}$.

Uma possível estratégia para aumentar a efetividade de protocolos de triagem seria focar em populações de risco, dentre as quais as de raça negra, com pressão intraocular acima dos níveis estatisticamente aceitos e parentes de glaucomatosos.

O HRTII apresenta boa reprodutibilidade das medidas já vastamente relatada na literatura ${ }^{(9)}$.Uma limitação no HRTII tem sido a sua dependência do operador em delimitar a margem do disco antes que os parâmetros topográficos possam ser calculados. No presente trabalho, o exame foi realizado pelo mesmo examinador e respeitado o desvio padrão das imagens em valores inferiores a $30 \mu \mathrm{m}$.

O presente trabalho corrobora a hipótese de que a herança familiar é um forte fator de risco para o glaucoma, estendendo-se às características morfométricas do disco óptico avaliadas pelo HRTII. Verificou-se que os parentes de primeiro grau de portadores da doença (grupo1) apresentaram discos ópticos maiores, quando comparados aos outros grupos. Quando comparados ao grupo controle, a área da escavação $(p=0,006)$, a relação $E / D(p=0,006)$ e a relação $E / D$ linear $(p=0,016)$ também foram maiores. Um estudo genético da relação E/D entre parentes de primeiro grau relatou correlação altamente sugestiva de herança poligênica multifatorial ${ }^{(10)}$. Mais recentemente, Chang et al. consideraram que a relação E/D em parentes de glaucomatosos era 0,07 (19\%) maior que em parentes de probandos normais $(\mathrm{p}=0,004)^{(11)}$.

Existe controvérsia se discos ópticos maiores correlacionam-se com maior risco de dano glaucomatoso. Fatores considerados favoráveis a essa teoria incluiriam: discos maiores e maior susceptibilidade ao dano 
glaucomatoso da população afro-americana quando comparada aos caucasianos, fatores mecânicos como o gradiente pressórico contra a lâmina crivosa produzindo maior deslocamento em discos maiores, susceptibilidade para perda do anel neuroretiniano em regiões com uma distância maior da artéria central da retina ${ }^{(12)}$. Porém, fatores contrários também são citados na literatura, como o fato de discos ópticos menores conterem menos fibras nervosas gerando menor reserva anatômica no caso de um eventual dano ${ }^{(12)}$.

Diante do exposto, considera-se que fatores favoráveis podem compensar os contrários. Diversos estudos concluíram que a área do disco óptico pode ser considerada um fator preditivo fraco para glaucoma de ângulo aberto ${ }^{(12)}$.

As diferenças observadas entre o grupo 1 e o grupo 3 reafirmam a noção de que um aumento na relação E/D em parentes próximos de portadores de glaucoma, possa ser realmente um traço herdado mais que puramente adquirido. Entretanto, sabe-se que a pressão intraocular permanece como importante fator de risco para a doença, também com hereditariedade estimada em 0,29 com diferença média de $1,02 \mathrm{mmHg}(\mathrm{p}=0,017)$ para mais em parentes de probandos com a doença ${ }^{(11)}$. Porém, não encontramos diferenças estatisticamente significantes para a pressão intraocular e espessura corneana central entre os grupos, fato esse justificado talvez pelo tamanho reduzido da amostra. Estudos prospectivos com maior número de famílias são necessários para corroborar nossos achados e estratificar a amostra por grupos etários definidos.

\section{Conclusões}

Parentes, principalmente de primeiro grau (pais ou irmãos), de portadores de glaucoma primário de ângulo aberto apresentam discos ópticos, áreas da escavação, relação E/D e relação E/D linear topograficamente maiores, quando comparados ao grupo controle. Nessas famílias, aconselhamos propedêutica de glaucoma em parentes adultos. Esta conduta permitirá a detecção precoce do glaucoma e o tratamento de indivíduos com alto risco.

\section{ReferêNCIAS}

1. Sociedade Brasileira de Glaucoma, Mello PAA, Mandia Júnior C, editores. II Consenso Brasileiro de Glaucoma Primário de Ângulo Aberto. São Paulo: PlanMark; 2005.

2. Quigley H. How common is glaucoma worldwide? Int Glaucoma Rev. 2002;3(3):11-2.

3. Wu J, Hewitt AW, Green CM, Ring MA, McCartney PJ, Craig JE, Mackey DA. Disease severity of familial glaucoma compared with sporadic glaucoma. Arch Ophthalmol. 2006;124(7):950-4.

4. Alward WLM. Os pacientes com glaucoma devem se submeter a um estudo genético? In: Susanna Júnior R, Weinreb RN. Glaucoma: perguntas e respostas. Rio de Janeiro: Cultura Médica; 2005. p.35-38.

5. Wolfs RC, Klaver CC, Ramrattan RS, van Duijn CM, Hofman A, de Jong PT. Genetic risk of primary open-angle glaucoma. Population-based familial aggregation study. Arch Ophthalmol. 1998;116(12):1640-5.

6. Klein BE, Klein R, Lee KE. Heritability of risk factors for primary open-angle glaucoma: the Beaver Dam Eye Study. Invest Ophthalmol Vis Sci. 2004;45(1):59-62.

7. Strouthidis NG, Garway-Heath DF. New developments in Heidelberg retina tomography for glaucoma. Curr Opin Ophthalmol. 2008;19(2):141-8.

8. Vegini F, Figueiroa Filho N, Lenci RF, Garcia Neto D, Susanna Junior R. Prevalence of open angle glaucoma in accompanying first degree relatives of patients with glaucoma. Clinics (Sao Paulo). 2008;63(3):329-32.

9. Vessani RM. Comparação entre diversas técnicas de imagem para diagnóstico do glaucoma [tese]. São Paulo: Faculdade de Medicina da Universidade de São Paulo; 2007.

10. Armaly MF. Genetic determination of cup/disc ratio of the optic nerve. Arch Ophthalmol. 1967;78(1):35-43.

11. Chang TC, Congdon NG, Wojciechowski R, Muñoz B, Gilbert D, Chen P, et al. Determinants and heritability of intraocular pressure and cup-to-disc ratio in a defined older population. Ophthalmology. 2005;112(7):1186-91.

12. Jonas JB, Martus P, Horn FK, Jünemann A, Korth M, Budde WM. Predictive factors of the optic nerve head for development or progression of glaucomatous visual field loss. Invest Ophthalmol Vis Sci. 2004;45(8):2613-8.

\section{Endereço para correpondência:}

Alinne Maria Camargos da Costa

R.Pernambuco, $\mathrm{n}^{\circ} 881 / 801$

CEP 30130-151 - Belo Horizonte (MG),Brasil

E-mail: alinnecamargos@gmail.com 